PROCEEDINGS OF THE

AMERICAN MATHEMATICAL SOCIETY

Volume 126, Number 2, February 1998, Pages 349-352

S $0002-9939(98) 04074-\mathrm{X}$

\title{
A REMARK ON GELFAND-KIRILLOV DIMENSION
}

\author{
S. PAUL SMITH AND JAMES J. ZHANG \\ (Communicated by Lance W. Small)
}

\begin{abstract}
Let $A$ be a finitely generated non-PI Ore domain and $Q$ the quotient division algebra of $A$. If $C$ is the center of $Q$, then $\operatorname{GKdim} C \leq$ GKdim $A-2$.
\end{abstract}

Throughout $k$ is a commutative field and $\operatorname{dim}_{k}$ is the dimension of a $k$-vector space. Let $A$ be a $k$-algebra and $M$ a right $A$-module. The Gelfand-Kirillov dimension of $M$ is

$$
\operatorname{GKdim} M=\sup _{V, M_{0}} \varlimsup_{n \rightarrow \infty} \log _{n} \operatorname{dim}_{k} M_{0} V^{n}
$$

where the supremum is taken over all finite dimensional subspaces $V \subset A$ and $M_{0} \subset M$. If $F \supset k$ is another central subfield of $A$, we may also consider the Gelfand-Kirillov dimension of $M$ over $F$ which will be denoted by GKdim $_{F}$ to indicate the change of the field. We refer to $[\mathrm{BK}],[\mathrm{GK}]$ and $[\mathrm{KL}]$ for more details.

Let $Z$ be a central subdomain of $A$. Then $A$ is localizable over $Z$ and the localization is denoted by $A_{Z}$. For any right $A$-module $M, M \otimes A_{Z}$ is denoted by $M_{Z}$. Let $F$ be the quotient field of $Z$. The first author [Sm, 2.7] proved the following theorem:

Let $A$ be an almost commutative algebra and $Z$ a central subdomain. Suppose $M$ is a right $A$-module such that $M_{Z} \neq 0$. Then

$\operatorname{GKdim} M \geq \operatorname{GKdim}_{F} M_{Z}+\operatorname{GKdim} Z$.

As a consequence of this, if $A$ is almost commutative but non-PI and $Z$ is a central subalgebra such that every nonzero element in $Z$ is regular in $A$, then $\operatorname{GKdim} Z \leq \operatorname{GKdim} A-2$.

It is natural to ask if the above theorem (and hence the consequence) is true for all algebras. In this paper we will precisely prove this.

Theorem 1. Let $A$ be an algebra and $Z$ a central subdomain. Suppose $M$ is a right $A$-module such that $M_{Z} \neq 0$. Then

$\operatorname{GKdim} M \geq \operatorname{GKdim}_{F} M_{Z}+\operatorname{GKdim} Z$.

An algebra is called locally PI if every finitely generated subalgebra is PI. As a consequence of Theorem 1 , we have

Received by the editors July 12, 1996 and, in revised form, August 20, 1996.

1991 Mathematics Subject Classification. Primary 16P90.

Key words and phrases. Gelfand-Kirillov dimension.

This research was supported in part by the NSF. 
Corollary 2. Let $A$ be algebra and $Z$ a central subdomain. If $A_{Z}$ is nonzero, then

$$
\operatorname{GKdim} A \geq \operatorname{GKdim}_{F} A_{Z}+\operatorname{GKdim} Z \text {. }
$$

Furthermore, if $A_{Z}$ is not locally PI, then

$$
\operatorname{GKdim} A \geq 2+\operatorname{GK} \operatorname{dim} Z \text {. }
$$

For the second inequality in Corollary $2, Z$ need not be a domain. Let $Z$ be any central subalgebra of $A$ of finite GKdimension such that $A_{Z}$ is not locally PI. By the Noether normalization theorem, there is a subalgebra $Z_{1} \subset Z$ isomorphic to the polynomial ring on $d$ variables where $d=\operatorname{GKdim} Z$. Since $A_{Z}=\left(A_{Z_{1}}\right)_{Z}, A_{Z_{1}}$ is nonzero and not locally PI. Hence, by Corollary $2, \operatorname{GKdim} Z_{1} \leq \operatorname{GKdim} A-2$. Therefore $\operatorname{GKdim} Z=\operatorname{GKdim} Z_{1} \leq \operatorname{GKdim} A-2$.

A stronger version of Corollary 2 also holds. We need another invariant defined by Gelfand and Kirillov. Let $A$ be an algebra. The Gelfand-Kirillov transcendence degree of $A$ is

$$
\operatorname{Tdeg} A=\sup _{V} \inf _{b} \operatorname{GKdim} k[b V]
$$

where $V$ ranges over all finite dimensional subspaces of $A$ and $b$ ranges over the regular elements of $A$. If $A$ is a commutative domain, then both GKdim $A$ and Tdeg $A$ are equal to the classical transcendence degree of $A$, denoted by $\operatorname{trdeg} A$. If $F \supset k$ is a central field of $A$, the Gelfand-Kirillov transcendence degree of $A$ over $F$ will be denoted by $\operatorname{Tdeg}_{F}$ to indicate the change of the field.

Theorem 3. Let $A$ be a semiprime Goldie algebra and $Q$ the classical quotient algebra of $A$. Let $F$ be a central subfield of $Q$. Then

$$
\operatorname{Tdeg} Q \geq \operatorname{Tdeg}_{F} Q+\operatorname{trdeg} F \text {. }
$$

If moreover $A$ is not locally PI, then

$$
\operatorname{GKdim} A \geq 2+\operatorname{GKdim} F .
$$

The statement in the abstract is an obvious consequence of Theorem 3 .

We now give the proofs. For simplicity a subspace means a finite dimensional subspace over $k$ and a subframe of an algebra means a subspace containing the identity. Our proofs are based on the following easy observation.

Lemma 4. Let $F \supset k$ be a commutative field and $M$ a right $F$-module. Let $M_{0} \subset$ $M$ and $W \subset F$ be subspaces over $k$. Then

$$
\operatorname{dim}_{k} M_{0} W \geq\left(\operatorname{dim}_{F} M_{0} F\right)\left(\operatorname{dim}_{k} W\right) .
$$

Proof. Pick a basis of $M_{0} F$ over $F$, say $\left\{x_{1}, \cdots, x_{p}\right\} \subset M_{0}$. Then $M_{0} F=\oplus_{i=1}^{p} x_{i} F$ and hence $M_{0} W \supset \oplus_{i=1}^{p} x_{i} W$. Therefore $\operatorname{dim}_{k} M_{0} W \geq\left(\operatorname{dim}_{F} M_{0} F\right)\left(\operatorname{dim}_{k} W\right)$.

Proof of Theorem 1. Since $Z$ is central, by the proof of [KL, 4.2], we have GKdim $M$ $\geq \operatorname{GKdim} M_{Z}$. By [KL, 4.2], GKdim $Z=\operatorname{GKdim} F$ where $F$ is the quotient field of $Z$. Hence it suffices to show GKdim $M_{Z} \geq \operatorname{GKdim}_{F} M_{Z}+\operatorname{GKdim} F$. Therefore we may assume $Z=F$ is a central field of $A$, and we need to show that GKdim $M \geq$ $\operatorname{GKdim}_{F} M+\operatorname{GKdim} F$. Let $d$ be any number less than GKdim $F$. Then there exists a subframe $S \subset F$ such that $\operatorname{dim}_{k} S^{n} \geq n^{d}$ for all $n \gg 0$. Let $e$ be any number less than $\operatorname{GKdim}_{F} M$. Then there exist a subspace $M_{0} \subset M$, and a subframe $V \subset A$ 
such that $\operatorname{dim}_{F} M_{0} F(V F)^{n} \geq n^{e}$ for infinitely many $n$. Since $A \supset F$, we may assume $V \supset S$. Since $F$ is central, $M_{0} F(V F)^{n}=M_{0} V^{n} F$. By Lemma 4 ,

$$
\operatorname{dim}_{k} M_{0} V^{2 n} \geq \operatorname{dim}_{k} M_{0} V^{n} S^{n} \geq\left(\operatorname{dim}_{F} M_{0} V^{n} F\right)\left(\operatorname{dim}_{k} S^{n}\right) \geq n^{e} n^{d}=n^{e+d}
$$

for infinitely many $n$. Hence $\operatorname{GKdim} M \geq e+d$. By the choices of $e$ and $d$, we obtain $\operatorname{GKdim} M \geq \operatorname{GKdim}_{F} M+\operatorname{GKdim} F$ as desired.

Proof of Corollary 2. The first inequality follows from Theorem 1.1 by letting $M=$ $A$. If $A_{Z}$ is not locally PI, then $\mathrm{GKdim}_{F} A_{Z}>1$ by [SSW], and $\mathrm{GKdim}_{F} A_{Z} \geq 2$ by $[\mathrm{Be}]$. Hence the second inequality follows.

As pointed out in [Sm, p. 37] the inequalities in Corollary 2 may be strict even if $Z$ is the maximal central subring. By a result of M. Lorenz [Lo] the same example in [Sm, p. 37] shows also that the inequalities in Theorem 3 may be strict. The proof of Theorem 3 is similar to that of Theorem 1.

Proof of Theorem 3. Since $F$ is commutative, for any $d<\operatorname{trdeg} F(=\operatorname{GKdim} F)$, there is a subframe $S \subset F$ such that $\operatorname{dim}_{k} S^{n} \geq n^{d}$ for all $n \gg 0$. Let $e$ be any number less than $\operatorname{Tdeg}_{F} Q$. By the proof of [Zh, 3.1] there is a subframe $V \subset A$ such that for every regular element $b \in Q, \operatorname{GKdim} F[b V F]>e$. This is equivalent to saying that, for every regular element $b \in Q, \operatorname{dim}_{F}(F+b V F)^{n} \geq n^{e}$ for infinitely many $n$. We may assume $V \supset S$. Since $F$ is central, $\operatorname{dim}_{F}(k+b V)^{n} b^{n} F=$ $\operatorname{dim}_{F}(F+b V F)^{n}$. By Lemma 4,

$$
\operatorname{dim}_{k}(k+b V)^{n}(b S)^{n} \geq\left(\operatorname{dim}_{F}(F+b V F)^{n}\right)\left(\operatorname{dim}_{k} S^{n}\right) .
$$

Hence

$$
\operatorname{dim}_{k}(k+b V)^{2 n} \geq \operatorname{dim}_{k}(k+b V)^{n}(b S)^{n} \geq n^{e} n^{d}=n^{e+d}
$$

for infinitely many $n$. This means that GKdim $k[b V] \geq e+d$ and hence $\operatorname{Tdeg} Q \geq$ $e+d$. By the choices of $e$ and $d, \operatorname{Tdeg} Q \geq \operatorname{Tdeg}_{F} Q+\operatorname{trdeg} F$.

Now we assume $A$ is not locally PI. Then $Q$ is not locally PI. By [SSW] and [Be], $\operatorname{GKdim}_{F} Q \geq 2$ and by [Zh, 4.1 and 4.3], $\operatorname{Tdeg}_{F} Q \geq 2$. Therefore by [Zh, 2.1 and $3.1]$

$\operatorname{GKdim} A \geq \operatorname{Tdeg} A \geq \operatorname{Tdeg} Q \geq \operatorname{Tdeg}_{F} Q+\operatorname{trdeg} F \geq 2+\operatorname{GKdim} F$.

If $Z$ is a central subdomian of $A$, we can similarly prove that $\operatorname{Tdeg} A \geq \operatorname{Tdeg}_{F} A_{Z}$ $+\operatorname{trdeg} Z$ where $F$ is the quotient field of $Z$.

\section{REFERENCES}

[Be] G. M. Bergman, A note on growth functions of algebras and semigroups, mimeographed notes, University of California, Berkeley, 1978.

[BK] W. Borho and H. Kraft, Über die Gelfand-Kirillov-Dimension, Math. Annalen 220 (1976), 1-24. MR 54:367

[GK] I. M. Gelfand and A. A. Kirillov, Sur les corps liés aux algèbres enveloppantes des algèbres de Lie, Publ. Math. I.H.E.S. 31 (1966), 5-19. MR 34:7731

[KL] G. Krause and T. H. Lenagan, Growth of algebras and Gelfand-Kirillov dimension, Research Notes in Mathematics, Pitman Adv. Publ. Program, vol 116, 1985. MR 86g:16001

[Lo] M. Lorenz, On the transcendence degree of group algebras of nilpotent groups, Glasgow Math. J. 25 (1984), 167-174. MR 86c:16005

[SSW] L. W. Small, J. T. Stafford and R. B. Warfield, Affine algebras of Gelfand-Kirillov dimension one are PI, Math. Proc. Camb. Phil. Soc. 97 (1985), 407-414. MR 86g:16025 
[Sm] S. P. Smith, Central localization and Gelfand-Kirillov dimension, Israel J. Math. 46 (1983), 33-39. MR 85k:16048

[Zh] J. J. Zhang, On Gelfand-Kirillov transcendence degree,, Trans. Amer. Math. Soc. 348 (1996), 2867-2899. MR 97a:16016

Department of Mathematics, Box 354350, University of Washington, Seattle, WashINGTON 98195

E-mail address: smith@math.washington.edu

E-mail address: zhang@math.washington.edu 